\title{
La valoración en matemáticas de la educación secundaria a la universitaria, perspectivas docentes
}

Luis Fernando Mariño ${ }^{1}$

\section{Resumen}

Intentando buscar alternativas diferentes a culpar siempre al estudiante acerca de la problemática que suscita el bajo rendimiento en matemáticas iniciando su pregrado, la educación media y pruebas estandarizadas aplicadas por el estado, se realizó una investigación de campo con una muestra no probabilística con el objetivo de analizar y contrastar los principios que orientan a un grupo de docentes de los dos últimos años de colegios públicos y Universidad Francisco de Paula Santander organizando la evaluación del conocimiento matemático en la ciudad de Cúcuta durante el año 2008. El trabajo se orientó por los acercamientos teóricos de Web (1992), quien intenta construir una teoría para la valoración en matemáticas, los instrumentos fueron el cuestionario y la encuesta estructurada. Entre los principales hallazgos se pueden citar: Si bien casi todos los docentes coinciden en que la matemática debe enseñarse como un cuerpo estructurado de conocimientos, esto difiere respecto a sus opiniones sobre la forma como los estudiantes aprenden matemáticas y las estrategias aplicadas en el proceso de enseñanza. Acerca de los principios que los orientan organizando actividades de evaluación, consideran posible otras formas diferente a la prueba escrita (un proyecto a lo largo de varios días o la entrevista), el rol que juega el docente al momento de aplicar pruebas, al instante de analizar y presentar información debe haber alternativas distintas a la emisión de un sólo veredicto sea cuantitativamente o cualitativamente, estipulan también que la valoración debe ser un proceso continuo y no terminal en la enseñanza y aprendizaje de esta área.

Palabras claves: valoración, principios, perspectivas.

\section{Abstract}

Trying to find alternatives to always blame the students about the problems raised by the poor performance in Mathematics when they begin their undergraduate school and they had standard tests performed by the State, we did a field research with a probabilistic sample trying to analyze and contrast the principles guiding a group of teachers in the last two years of public schools and the University Francisco de Paula Santander, we organized the assessment of mathematical knowledge in Cucuta in 2008. The research was guided by the theoretical approaches to Web (1992), who tries to construct a theory for the assessment in Mathematics; the instruments were the questionnaire and the structured

${ }^{1}$ Docente Departamento de Matemáticas y Estadística UFPS, fermarin3@gmail.com 
survey. Among the main findings can be mentioned: although most teachers agree that Math should be taught as a formal body of knowledge, this is different regarding their views on how students learn Mathematics and the strategies applied in the process of teaching. About organizing principles that guide evaluation activities, teachers consider different possible forms of written test (a project over several days or interview), the role played by teachers when applying tests during analysis and presentation of information must have alternatives to the issuance of a just result is quantitatively or qualitatively, also provide that the valuation should be an ongoing process and not subsequent in the teaching and learning in this area.

Keywords: assessment, principles, perspectives

\section{Introducción}

Un estudio realizado en Colombia a 70 instituciones permitió estimar, en el caso de las universidades, una deserción por cohortes del $48.2 \%$ para el periodo 1998-2004, la mayor tasa fue de 23,5\%, correspondiente al paso entre el primero y segundo semestre, uno de los factores más influyente es el rendimiento académico (Revista Educación Superior en Colombia, 2006). El bajo rendimiento en matemáticas en los primeros semestres universitarios, en educación secundaria y las pruebas estandarizadas a nivel nacional presentadas finalizando el bachillerato por quienes aspiran ingresar a la educación superior, genera discrepancias entre directivos y docentes de estos niveles educativos quienes se culpan mutuamente por estas circunstancias.

Según Gil, F., y otros, (1993) el sistema de evaluación en matemáticas se caracteriza por: la temporalización estricta del sistema limitando en una, dos o hasta cuatro pruebas escritas cada periodo académico, la entrega final de una calificación resumiendo en un solo dato o frase la complejidad del aprendizaje logrado por el alumno, sin valoran errores ni preguntas no contestadas, el nivel de dominio aceptable se establece arbitrariamente y en colegios se obliga a recuperaciones en fechas determinadas. En Colombia cada institución evalúa el aprendizaje de sus estudiantes en forma diferente, mientras la mayoría de universidades tienen un sistema cuantitativo con escala entre cero y cinco, el sistema de educación precedente lo hace de forma cualitativa, sea por logros, indicadores de logro, estándares, competencias etc.; y según el Decreto 230 (2002), cada institución debe ga- rantizar un mínimo de promoción del 95\% de los estudiantes finalizando el año.

Ante esta problemática y buscando generar alternativas diferentes a la de buscar causas y explicaciones en el estudiante, se llevó a cabo un estudio centrado en las perspectivas de un grupo de docentes cuando diseñan y organizan actividades de evaluación de sus estudiantes; se propuso como objetivo general: Analizar diferencias o similitudes acerca de los principios que orientan a docentes de los últimos dos años de educación secundaria en colegios públicos y la Universidad Francisco de Paula Santander (UFPS), organizando la valoración en matemáticas en la ciudad de Cúcuta durante el año 2008.

La investigación se orientó por los acercamientos teóricos propuestos por Webb (1992), quien intenta construir una teoría de la valoración en matemáticas, para este autor la valoración en matemáticas es: "La consideración comprensiva del funcionamiento de un grupo o individuo en matemáticas o en la aplicación de las matemáticas". Considera tres principios básicos para organizar la evaluación del conocimiento matemático: especificar el contenido, formular la valoración y la valoración como parte integral de la instrucción. Especificar el contenido es el resultado de un propósito para la valoración, una concepción de las matemáticas y una teoría del aprendizaje de las matemáticas. La formulación de una valoración requiere considerar cuatro componentes generales del proceso: (a) la situación en la que se hace la valoración, (b) la respuesta a esta situación, (c) el análisis de la respuesta y (d) la interpretación de los resultados. Estos tres principios, junto con la planifica- 
ción y estrategias de enseñanza de la matemática dieron orientación y sentido al estudio.

\section{Metodología}

Estudio de campo exploratorio y descriptivo en un colectivo de docentes de matemáticas, la población estuvo conformada por 110 docentes: 51 de la Universidad Francisco de Paula Santander y 59 de los grados décimo y undécimo de colegios públicos de la ciudad de Cúcuta. La muestra no probabilística estuvo integrada por 69 profesores voluntarios: 28 de la UFPS que orientan materias de Cálculo Diferencial, Integral, y Multivariado, Ecuaciones Diferenciales, Métodos Numéricos, Algebra Lineal, Series, Geometría, Teoría de Números, Análisis Matemático y Estadística entre otras, 41 de los dos últimos grados en colegios públicos, algunos de ellos además de matemáticas, enseñan física o informática, otros matemáticas en grados precedentes, cumplen labores de jefes de área, titulatura de grupo o participan en grupos de investigación.

Las técnicas utilizadas para recolectar la información fueron la encuesta estructurada adhoc, diseñada específicamente con el propósito de poder describir características acerca de la forma como los integrantes de la muestra planifican la enseñanza de la matemática, sus estrategias, principios que los orientan organizando la valoración en matemáticas y algunas tendencias en sus prácticas reales de evaluación y un cuestionario para recolectar información sobre experiencia académica y nivel de estudios de los participantes.

Para el procesamiento y análisis la información se utilizó SPSS versión en español, como una finalidad era comparar posibles diferencias o similitudes en los dos grupos de docentes, para el análisis se optó por las tablas de contingencia (tablas de doble entrada), en lo que respecta a las características de los participantes se utilizaron algunos elementos de la estadística como: media, rango y percentiles, etc.

\section{Resultados y discusión}

Características del profesorado. Del total de integrantes de la muestra se observó que el 59,4\% son hombres, en tanto que el $34,1 \%$ de los docentes de colegios son mujeres (14 de 41), en cuanto a la edad por grupos se tiene: la edad promedio en docentes de colegio es 46,05 años (41,8 universidad), el docente más joven en colegios tiene 33 años (27 universidad), el de mayor edad es 58 años en colegios y 64 en universidad, por su parte el promedio de edad de la totalidad de los participantes es de 44,33 años.

En cuanto a nivel de quienes tienen postgrados se hallaron algunas diferencias como: el $88,5 \%$ de profesores tiene grado de especialización $(53,8 \%$ en colegios contra un $34,8 \%$ de la universidad), el porcentaje restante tiene maestría $(3,8 \%$ de colegios y $7,7 \%$ de universidad), sólo dos de la universidad tienen maestría en educación especialidad matemática.

En el factor experiencia a nivel global, se advierte que: aunque de la totalidad de participantes 55 de ellos tienen experiencia en grado décimo y 46 en undécimo el promedio de experiencia es muy similar (10,5 y 10,4 años respectivamente), el 75\% (de 55) tienen 15 o menos años de experiencia en grado décimo, en tanto que el 50\% (de 46) tienen experiencia de 8,5 años o menos en grado undécimo.

Un número bastante alto de integrantes de la muestra tiene experiencia en Cálculo Diferencial (46 de 69) e Integral (38) y su promedio es muy parejo (5,7 años y 5,1 respectivamente), a su vez la tercera parte de ellos tiene 7,75 años de experiencia o menos en estas materias; el 50\% de profesores de Cálculo en varias variables tiene 3 o menos años de experiencia en esta materia. El promedio de experiencia en materias como Ecuaciones Diferencial y Series es bastante bajo (5,83 y 5,5 años).

Planificación de la enseñanza de la matemática. El 39,1\% del profesorado está de acuerdo en que siempre al inicio de cada periodo académico se plantean metas y objetivos respecto a la enseñanza, el 23,2\% coincide en que se realiza casi siempre o algunas veces, no obstante considerando a los dos colectivos por separado la tercera parte 
del profesorado de secundaria afirma que siempre se hace, mientras que sólo el 5,8\% de sus colegas universitarios lo reconoce; una diferencia bastante amplia. Por su parte el 13,0\% de los docentes universitarios admite que nunca se realizan estas actividades.

Enseñanza de la matemática. El 56,7\% de los participantes afirman que los estudiantes aprenden matemáticas cuando a partir de destrezas simples aprenden secuencias de destrezas complejas, los demás opinan que aprenden cuando se logra alterar las estructuras mentales mediante el aprendizaje de conceptos; analizando por separado se notan diferencias entre los grupos, ya que el $37,7 \%$ de docentes de colegios están de acuerdo con la primera opción contra el 18,8\% de docentes universitarios.

La valoración en matemáticas. El 30,4\% de los docentes considera que el propósito de la valoración es utilizarla como herramienta para conseguir evidencia sobre lo que los estudiantes conocen y son capaz de hacer en matemáticas, el 15,9\% opina que debe ser un proceso de realimentación entre profesor-estudiantes, el 14,5\% de los profesores de colegios están de acuerdo con las dos anteriores, entretanto sólo el 4,3\% de ellos la opina que se debe emplear como sistema de información a la sociedad acerca de la efectividad del sistema educativo.

El 55,1\% opina que la enseñanza de la matemática debe hacer más énfasis en el desarrollo de habilidades, destrezas y formación de hábitos, mientras el 44,9\% dice que en el aprendizaje de conceptos. Sin embargo revisando separadamente se observaron diferencias ya que sólo el 10,1\% de profesores universitarios opina que se debe hacer énfasis en el desarrollo de habilidades, destrezas y formación de hábitos contra un 44,9\% de profesores de colegios.

Respecto a las formas de evaluar, analizando por grupos, se aprecian algunas diferencias: el 24,6\% de los profesores de colegios considera posible un proyecto a lo largo de varios días como forma de valorar, mientras qué sólo el 7,2\% de profesores universitarios está de acuerdo en esto, el 4,3\% de los docentes de colegios piensan en la entrevista como alternativa, en la universidad ninguno, por otro lado entre quienes piensan que un proyecto a lo largo de varios días y una pregunta en clase como posibilidades para evaluar, el porcentaje de docentes universitario $(11,6 \%)$ duplica al de sus colegas de colegios, aproximadamente la tercera parte de los profesores (31,9\%) está de acuerdo en que un proyecto a lo largo de varios días es una muy buena opción para evaluar en matemáticas.

Un poco más de la mitad $(52,2 \%)$ de docentes de colegios considera que la estructura y la forma en que se presenta la evaluación a los estudiantes influye en los resultados de ésta, en tanto que la tercera parte de sus colegas universitarios parece estar de acuerdo, en cuanto a la totalidad se observa que sólo el $14,5 \%$ de los participantes afirman que esto no influye en los resultados.

Un porcentaje bastante alto $(95,7 \%)$ de los participantes considera acertado el uso problemas abiertos como preguntas de evaluación; donde se proporcionen datos y condiciones específicas (situaciones problema donde inclusive no se conoce la solución), permitiendo que los estudiantes hagan sus interpretaciones, propongan soluciones, justifiquen y expliquen; analizando separadamente el 58,0\% de los profesores de los colegios está de acuerdo con esta opción contra un $37,7 \%$ de docentes de universidad.

Se apreciaron discrepancias entre grupos respecto a si al momento de analizar la respuesta de un estudiante ante una pregunta de evaluación, se debe emitir un juicio correcto o incorrecto o estimar si el estudiante está preparado para aprender una estrategia más compleja puesto que el 53,4\% de docentes de colegios está de acuerdo en que al momento de analizar la respuesta se debe estimar si el estudiante está preparado para aprender una estrategia más compleja contra el 30,4\% de los profesores de la universidad.

El 71,0\% parece estar de acuerdo en qué después del proceso de evaluación es conveniente juzgar al estudiante sobre el progreso realizado a partir de lo que conocía al comienzo de la instrucción, no obstante analizando separadamente a los grupos se perciben diferencias: el $44,9 \%$ del grupo de secundaria considera que se debe juzgar al estudiante por el progreso en el conocimiento contra el $26,1 \%$ de universitarios, en cuanto a si se debe comparar al estudiante respecto al grupo u otra institución un porcentaje bajo (14,5\%) afirma que sí. 
Actividades reales de evaluación. Un porcentaje alto de profesores afirma que sus índices de reprobación en el último semestre o año académico estuvo entre el $40 \%$ y $60 \%$, al preguntar sí: iLas estrategias, actividades y recursos de enseñanza, no estuvieron acorde en cuanto a contenidos, tiempo y espacio, no logran motivar al estudiante u otra razón? Se presentaron algunas diferencias como: el 33,3\% de docentes de colegios admite que las estrategias, actividades y recursos no logran motivar al estudiante, factor que incide en el rendimiento académico, entretanto sólo el 14,5\% de sus colegas de la universidad lo reconoce, un $15,9 \%$ de la universidad acepta que no fueron acordes en cuanto a contenidos, tiempo y espacio contra un $20,3 \%$ de docentes de colegios.

Se observó que el 39,1\% de los participantes acepta que sus preguntas de evaluación provienen de textos guía, entretanto el $43,5 \%$ de ellos dice que las crean de acuerdo a su experiencia, y el 8,7\% declara que las obtiene de Internet, mientras un porcentaje muy bajo afirma que las genera con sus estudiantes en aula de clase, se aprecian discrepancias debido a que el 30,4\% del profesorado de colegios afirma que las preguntas las generan de acuerdo a la experiencia, en tanto que sólo el 13,0\% de universidad lo hace, también se advierte similitudes: igual porcentaje de ambos grupos reconoce que las crea, las obtiene de Internet o de las pruebas ICFES.

El 81,2\% está de acuerdo en diseñar y ejecutar actividades de refuerzo y recuperación, el 5,8\% de los participantes afirma que permite a los estudiantes corregir errores haciendo seguimiento, un docente universitario confirma que asesora individualmente durante el semestre. El 56,5\% de docentes de secundaria diseña y ejecuta estas actividades, entretanto sólo el 24,6\% de universidad lo hace, por otro lado el $13 \%$ de ellos opina que no hace nada al respecto, ninguno de sus colegas de colegio está de acuerdo en esto.

Cuando gran porcentaje del grupo reprueba una evaluación de matemáticas, el 31,9\% de participantes de colegios replantea si es necesario sus actividades en cuanto a contenidos, estrategias de enseñanza y procesos de evaluación cuando esto ocurre, en cambio sólo el 23,2\% de docentes universitarios lo hace, por su parte el $42,0 \%$ afirma que ante esta situación identifica posibles dificultades de aprendizaje o motivación del estudiante, reorientando el proceso de enseñanza, únicamente el $2,9 \%$ de ellos opina que realiza con el grupo actividades autoevaluación y coevaluación para mejorar.

Si bien casi todos los docentes coinciden en que al inicio de cada periodo se plantean metas y objetivos respecto a la enseñanza-aprendizaje, y que la matemática debe enseñarse como un cuerpo estructurado de conocimientos, contradiciendo sus opiniones sobre la forma como los estudiantes aprenden matemáticas y las estrategias aplicadas en el proceso de enseñanza, la pregunta es iqué ocurre al respecto?, ilos docentes utilizan los resultados de la evaluación como un proceso continuo y no terminal?, iqué ocurre en los procesos de evaluación y autoevaluación que se realiza en los departamentos de matemáticas de cada institución?.

\section{Conclusiones}

Las instituciones de educación básica primaria, secundaria y media, se rigen hasta el momento por el Decreto 230 (2002), coincidiendo con la temporalidad de la elaboración de este informe el MEN emite el Decreto 1290 (2009), como marco para la evaluación del aprendizaje de los estudiantes, en él se da libertad a cada institución para que adopte su escala de valoración del desempeño en su sistema de evaluación, escala que debe ser equivalente con la escala de valoración nacional, expresada en términos de desempeño: superior, alto, básico y bajo, brinda también autonomía en el sistema de promoción, en tanto en la UFPS sólo se referencia la evaluación en sentido general, no existe normatividad ni fundamentos teóricos claros que orienten estas actividades, el sistema de calificación según Acuerdo 065 (1996) del Estatuto Estudiantil.

En los dos últimos años de secundaria las instituciones educativas brindan espacios y tiempos para planificar la enseñanza de la matemática al inicio del año académico e incluso al final, más no en el transcurso de éste, entre tanto en la UFPS muy pocas veces se hace. 
Los profesores integrantes de la muestra consideran que sus estrategias de enseñanza siempre están orientadas por las teorías constructivistas, buscando activar conocimientos previos, trabajo de grupo colaborativo, resolución de problemas, etc. afirmación contraria a la forma como según ellos los alumnos aprenden matemáticas.

Si bien casi todos los docentes coinciden en que la matemática debe enseñarse como un cuerpo estructurado de conocimientos, esto difiere respecto a sus opiniones sobre la forma como sus estudiantes aprenden matemáticas y las estrategias aplicadas en el proceso de enseñanza.

Acerca de los principios que los orientan para organizar la valoración del conocimiento de sus estudiantes consideran posibles otras formas diferentes a la prueba escrita, el rol del docente al momento de aplicar las pruebas, al instante de analizar y presentar información del aprendizaje del estudiante debe haber otras alternativas diferentes a la emisión de un sólo veredicto, estipulan también que la valoración debe ser un proceso continuo y no final en el proceso enseñanza-aprendizaje.

A modo de conclusión final el autor considera que una vez se confirma lo hallado en muchos estudios de diferente tipo: el distanciamiento entre la teoría y la práctica, ya que los docentes tienen sus perspectivas y consideraciones acerca de lo que podrían hacer valorando el conocimiento matemático de sus estudiantes pero otra cosa es lo que realmente hacen, más relevante aún las discrepancias entre los dos niveles educativos respecto al tema en cuestión.

\section{Bibliografía}

Estatuto Estudiantil Universidad Francisco de Paula Santander (Acuerdo No. 065). (1996, Agosto 26). [Trascripción en línea]. Disponible: http://www.ufps.edu.co/ufpsnuevo/menu/pdf/ acuerdo065.pdf [Consulta, 2009, Mayo 11].

García, G. (2003). Currículo y Evaluación en Matemáticas. Un Estudio en Tres Décadas de Cambio en la Educación Básica. (1ª ed.). Bogotá: Editorial Magisterio.

Gil F., Castro E., Rico L. (1993). La evaluación en matemáticas: Revisión y estado de la cuestión. Ponencia presentada en VI Jornadas Andaluzas de Educación Matemática (pp 205-225). Sevilla: Sociedad Andaluza de Educación Matemática Thales. [Ponencia en línea]. Consultado el 15 de octubre de 2007 en: http://cumbia.ath.cx:591/pna/ Archivos/CastroE93-2686.PDF.

Hernández, S., Fernández, C. y Baptista, P. (1998). Metodología de la Investigación. ( ${ }^{\mathrm{a}}$ ed.). México: McGraw-Hill.

Ministerio de Educación Nacional. Revista Educación Superior. Diagnóstico de la Deserción estudiantil. [Revista en línea]. Disponible en: http:// menweb.mineducacion.gov.co/educacion_superior/numero_07/001.htm.

Normas en materia de currículo, evaluación y promoción de los educandos y evaluación institucional (Decreto No. 230). (2002, Febrero 11). [Trascripción en línea]. Disponible: http://www. dafp.gov.co/leyes/D0230002.HTM [Consulta, 2009, Mayo 11].

Reglamentación de la evaluación del aprendizaje y promoción de los estudiantes de los niveles de educación básica y media. (Decreto No. 1290). (2009, Abril 16). [Trascripción en línea]. Disponible: http://www.mineducacion.gov.co/1621/ articles-187765_archivo_pdf_decreto_1290.pdf [Consulta, 2009, Mayo 11].

SECRETARIA DE EDUCACIÓN MUNICIPAL CUCUTA. (2007). Subsecretaría de Planeación y Desarrollo Educativo. Cúcuta.

Webb, N. L. (1992). Traducción (de Rico, L. 1996). resumida del capitulo Assessment of Students' Knowledge of Mathematics: Steps toward a Theory. En Grouws, D.A. (Ed.)Handbook of Research on Mathematics Teaching and Learning. New York: Macmillan, pp. 661-683. [Traducción en línea], Consultado el 27 de septiembre de 2007 en: http://cumbia.ath.cx:591/pna/Archivos/RicoL96-109.PDF 